\title{
Miller-Fisher Syndrome following the first dose of Comirnaty (Pfizer COVID-19 vaccination)
}

\section{Pierre-Yves Sansen ${ }^{1}$, Edita Calaras ${ }^{2}$, Nicolas Weynants ${ }^{1}$, Philippe Glorieux ${ }^{1 *}$}

${ }^{1}$ Department of Internal Medicine, Cliniques du Sud-Luxembourg (Vivalia), Arlon, Belgium.

${ }^{2}$ Department of Neurology, Cliniques du Sud-Luxembourg (Vivalia), Arlon, Belgium.

*Corresponding Author: Philippe Glorieux, Department of Internal Medicine, Cliniques du Sud-Luxembourg (Vivalia), Arlon, Belgium.

Received date: 14 November 2021; Accepted date: 23 November 2021; Published date: 29 November 2021

Citation: Sansen PY, Calaras E, Weynants N, Glorieux P (2021) Miller-Fisher Syndrome following the first dose of Comirnaty (Pfizer COVID19 vaccination). J Med Case Rep Case Series 2(16): https://doi.org/10.38207/JMCRCS/2021/0216234

Copyright: (C) 2021 Philippe Glorieux. This is an open-access article distributed under the terms of the Creative Commons Attribution License, which permits unrestricted use, distribution, and reproduction in any medium, provided the original author and source are credited.

\begin{abstract}
A 65-year-old Caucasian man with type 2 diabetes mellitus presented to the emergency department with a two-day history of diplopia and ataxia. He received his first dose of the Comirnaty, the Pfizer COVID-19 vaccine, thirty-five days prior. Physical examination revealed ataxia with a left deviation of the body and a left VI nerve palsy demonstrated by an inability to move the left eye to the left. The patellar reflexes were abrogated at both sides, but the other reflexes were present. Cerebrospinal fluid analysis indicated an albuminocytologic dissociation consistent with GuillainBarré syndrome. Serum anti-sulfatide but not anti-GQ1B antibodies were found, further suggesting peripheral neuropathy, and negative serologic SARS-CoV2 testing excluded a past infection. Administration of intravenous immunoglobulins was successful, and the patient regained the ability to walk three weeks after therapy. We excluded typical causes of demyelinating disorders and concluded that COVID-19 mRNA vaccination can lead to Miller-Fisher syndrome. This case highlights the importance of considering Miller-Fisher syndrome as a possible, albeit rare, vaccineinduced side effect.
\end{abstract}

Keywords: Miller-Fisher syndrome, Guillain-Barré syndrome, demyelinating disorders, SARS-CoV2 mRNA vaccines.

\section{Introduction}

Miller-Fisher Syndrome (MFS) is a rare demyelinating disease that belongs to the Guillain- Barré group of neurological disorders. It is classically diagnosed in the presence of a triad of symptoms: ophthalmoplegia, ataxia, and areflexia [1]. Ophthalmologic involvement generally precedes other features, distinguishing MFS from other diseases such as myasthenia gravis, thyroid eye diseases, and myotonic dystrophy [4]. Most MFS patients demonstrate serum anti- GQ1b antibodies [2], which can block acetylcholine release from motor neurons and therefore induce the palsy that characterizes the syndrome. Other antibodies are also identified in patients, such as anti-sulfatide antibodies, but these are primarily associated with peripheral neuropathy [3].

\section{Case Presentation}

A 65-year-old male patient presented at the emergency with a twoday history of progressive diplopia and ataxia. Pre-existing comorbidities included type two diabetes mellitus and prostatic hyperplasia but no other known disease. He received a first dose of COVID-19 vaccination (Pfizer Comirnaty) 35 days before developing these symptoms. Physical examination revealed ataxia with a left deviation of the body and a left nerve VI palsy indicated by an inability to move the left eye to the left. The patellar reflexes were abrogated at both sides, while the other reflexes were present.
Approximately $72 \%$ of cases are associated with a past viral infection, generally within the previous ten days [1]; however, as in Guillain Barré syndrome (GBS), Campylobacter jejuni infection is also a common cause [6]. Vaccination-related cases have also been described, primarily following influenza vaccination, but these remain infrequent [7]. Although cases of GBS in association with COVID-19 mRNA vaccination have been previously described, none report Miller-Fisher syndrome [9]. However, an association between SARS-CoV2 infection and MFS is well-established [8].

The resolution of MFS may include intravenous immunoglobulin treatment, which appears to be efficient in MFS; however, because of the spontaneously favorable evolution of the disease, some studies show no impact on outcomes [5]. Thus, the prognosis is generally excellent with no remaining deficits [2]

A lumbar puncture was performed and showed albuminocytologic dissociation $(3$ elements $\mu \mathrm{L}$ [normal $<5$ elements $/ \mu \mathrm{L}])$, CSF protein $153 \mathrm{mg} / \mathrm{dL}$ [normal 30.0-50.0 mg/dL]). Microbiological investigations were negative. Medullary magnetic resonance imaging revealed no lesion. Analysis of anti-ganglioside antibodies permitted us to identify anti-sulfated $\operatorname{IgM}$, which remained positive on a second serologic evaluation one month later. Interestingly, an anti-GQ1b assay was negative, and negative SARS-CoV2 serology excluded the previous infection. 
Furthermore, our patient developed cytopenia composed of anemia and thrombocytopenia. Thrombotic microangiopathy was excluded due to the absence of schizocytes. Treatment with NSAIDs (diclofenac) was implicated as a cause of cytopenia. Although NSAIDs use is rarely implicated in thrombocytopenia and the patient received treatment with diclofenac ten days prior, a vaccinal cause was not formally excluded. Positive direct Coombs antiglobulin was further suggestive of an auto-immune process. Conversely, D-dimers and fibrinogen were both normal, which is uncommon in the case of vaccination-induced thrombocytopenia.

\section{Discussion}

MFS is a demyelinating disease that has been reported after SARSCoV2 infection [9]. It is diagnosed via a pathognomonic triad comprising ophthalmoplegia, ataxia, and areflexia. mRNA COVID19 vaccines, such as those received by our patient, have a potent capability to induce both humoral and cellular immunization, thereby efficiently protecting against SARS- CoV2 infection [12]. However, although stimulation of the immune system induces anti- SARSCoV2 spike protein antibodies, it can also predispose patients to the development of myelin-specific autoantibodies [11], potentially

\section{Conclusions}

We report a case of MFS following an mRNA COVID-19 vaccination (Pfizer Comirnaty). This article highlights the importance of a low threshold of suspicion for autoimmune neurological complications after mRNA vaccination, especially in the context of current mass

\section{References}

1. Berlit P, Rakicky J (1992) The Miller Fisher syndrome. Review of the literature. J Clin Neuroophthalmol. 12(1): 57-63.

2. Teener JW (2012) Miller Fisher's syndrome. Semin Neurol. 32(5): 512-6.

3. Giannotta C, Di Pietro D, Gallia F, Nobile-Orazio E (2015) Antisulfatide IgM antibodies in peripheral neuropathy: To test or not to test? Eur J Neurol. 22(5): 879-82.

4. Bukhari S, Taboada J (2017) A Case of Miller Fisher Syndrome and Literature Review. Cureus. 9(2): e1048.

5. Mori M, Kuwabara S, Fukutake T, Hattori T (2007) Intravenous immunoglobulin therapy for Miller Fisher syndrome. Neurology. 68 (14): 1144-1146.

6. Salloway S, Mermel LA, Seamans M, Aspinall GO, Nam Shin JE, et al. (1996) Miller-Fisher syndrome associated with Campylobacter jejuni bearing lipopolysaccharide molecules that mimic human ganglioside GD3. Infect Immun. 64(8): 29452949.
Unfortunately, an analysis for the presence of anti-platelet factor 4 antibodies by ELISA was not conducted.

A normal bone marrow biopsy excluded central involvement. Interestingly, anti-sulfatide antibodies have been described in sera of patients suffering from idiopathic thrombocytopenic purpura [10]. The patient was treated by a five-day immunoglobulin infusion $(0.4$ $\mathrm{g} / \mathrm{kg}$ ). The resolution was favorable, with significant improvement of ataxia and minor remaining ophthalmic sequelae within two weeks. The cytopenia improved and progressively resolved.

resulting in demyelinating autoimmune disorders. Interestingly, antisulfatide antibodies have been described in sera of patients suffering from idiopathic thrombocytopenic purpura [10].

However, these complications are not specific to the COVID-19 vaccination, and hundreds of publications report Guillain-Barré disorders after many types of vaccines [13]. Nonetheless, given the magnitude of the SARS-CoV2 pandemic, vaccination benefits clearly surpass these rare adverse effects.

vaccination efforts. Fortunately, the prognosis of MFS is excellent, and the benefits of vaccination surpass the risk of this rare complication.

7. Sanz Fadrique R, Martín Arias L, Molina-Guarneros JA, Jimeno Bulnes N, García Ortega P (2019) Guillain-Barré syndrome and influenza vaccines: current evidence. Rev Esp Quimioter. 32(4): 288-295.

8. Fernandez-Dominguez J, Ameijide-Sanluis E, Garcia-Cabo C, García-Rodríguez R, Mateos V (2020) Miller-Fisher-like syndrome related to SARS-CoV-2 infection (COVID 19). J Neurol. 267: 2495-2496.

9. Waheed S, Bayas A, Hindi F, Rizvi Z, Espinosa PS (2021) Neurological Complications of COVID-19: Guillain-Barre Syndrome Following Pfizer COVID-19 Vaccine. Cureus. 13(2): e13426.

10. van Vliet HH, Kappers-Klunne MC, van der Hel JW, Abels J (1987) Antibodies against glycosphingolipids in sera of patients with idiopathic thrombocytopenic purpura. British Journal of Haematology, 67(1): 103-108.

11. Haber P, Sejvar J, Mikaeloff Y, DeStefano F (2009) Vaccines and Guillain-Barré syndrome. Drug Saf. 32(4):309-23. 
12. Sadarangani M, Marchant A, Kollmann TR (2021) Immunological mechanisms of vaccine- induced protection against COVID-19 in humans. Nat Rev Immunol 21: 475-484.

13.

.
Principi N, Esposito S (2019) Vaccine-preventable diseases, vaccines and Guillain-Barre' syndrome. Vaccine. 37(37): 55445550 . 